\title{
The coverage of young children in demographic surveys
}

\author{
Eric B. Jensen and Howard R. Hogan \\ U.S. Census Bureau, Washington, DC 20233, USA
}

\begin{abstract}
The 2010 U.S. Decennial Census had a 4.6 percent net undercount for the population age 0 to 4 compared to a 0.1 percent over count for the total population. While the undercount of young children in the census has gotten considerable attention in recent years, less is known about the coverage of children in demographic surveys. In this paper, we analyze coverage rates by age, race, and Hispanic origin for three surveys conducted by the U.S. Census Bureau - American Community Survey (ACS), Current Population Survey (CPS), and the Survey of Income and Program Participation (SIPP). In addition, we estimate modified coverage rates to account for cumulative coverage error in both the survey and the census counts, which are used to calculate the coverage rates. The results show that young children tend to have lower coverage rates than other age groups. Coverage rates for young children in the ACS vary by race and Hispanic origin. The differences in coverage rates for young children in the CPS and SIPP by race and Hispanic origin were not statistically significant.
\end{abstract}

Keywords: Children, undercount, surveys, coverage rates

\section{Introduction}

The estimated net undercount for young children, age 0 to 4 , in the 2010 Census was 4.6 percent compared to a .01 over count for the total population [1]. The net coverage error for young children was even higher for some race and ethnic groups. The undercount of young children in the decennial census is a persistent problem that has been documented by demographers for many decades [2] (See for example, Coale 1955, Table 7). Recent estimates show that the net undercount of young children in the Census is driven by 2.2 million omissions for young children [3]. In other words, one out of every ten young children were not included in the 2010 Census.

In 2013, the Census Bureau organized a task force on the undercount of young children and a research team was created in 2015 to analyze this issue. While the focus of the task force and research team has been on the coverage of children in the census, we also

${ }^{*}$ Corresponding author: Eric B. Jensen, U.S. Census Bureau, 4600 Silver Hill Road, Washington, DC 20233, USA. Tel.: +1 301763 3723; E-mail: Eric.B.Jensen@census.gov. wanted to evaluate the coverage of this population in demographic surveys.

There are reasons we would expect demographic surveys to have patterns of coverage similar to the census and other reasons why we would expect demographic surveys to have different coverage patterns from the census. Some demographic surveys and the census ask respondents to create a roster of household members [4]. Surveys and the census both include operations to address nonresponse and increase overall participation. However, surveys have less extensive nonresponse followup and coverage improvement procedures, may have a panel or longitudinal design, are often collected throughout the year leading to seasonal variation in coverage, and do not have the same level of marketing and advertising as the decennial census.

Coverage in a survey is measured by calculating the relative coverage rate, which is the ratio of the uncontrolled survey estimate for a population to an independent population estimate. Many of the demographic surveys conducted by the Census Bureau use data from the Population Estimates Program as survey controls to adjust for coverage error. In other words, survey results are weighted to make sure they are consistent with 
the population estimates. The Census Bureau's Postcensal Population Estimates use the most recent census counts as the base population and then account for births, deaths, and migration since the census to provide current population estimates. The population estimates are produced by age, sex, race, and Hispanic origin. The coverage rates for the surveys can be calculated for these same characteristics, but not for additional characteristics such as poverty status or household structure.

In this report, we analyze coverage rates by age, race, and Hispanic origin for the American Community Survey (ACS), Current Population Survey (CPS), and the Survey of Income and Program Participation (SIPP). These surveys have very different sample designs, but are each controlled to the postcensal population estimates, which allows us to calculate relative coverage rates. As stated above, the population estimates are based on the decennial census, which undercounts young children. To address this issue, we use data from 2009 and from 2015, when available. In these years, the population age 0 to 4 would have been born after the census; therefore, the population estimates for this cohort were developed primarily from vital statistics data on births and not census counts. We then calculate adjusted coverage rates for the cohorts where the population estimates are based on the census to account for coverage error in the census. This allows more legitimate comparisons across age groups.

\section{Background}

The 2010 Census undercounted young children by an estimated 4.6 percent [1]. The Census Bureau measures coverage in the census using Demographic Analysis (DA) and dual-system estimation (DSE). DA uses historical vital statistics data on births and deaths and data on international migration to develop independent estimates of the population that are then compared to the census to measure coverage. The DSE approach - called Census Coverage Measurement (CCM) in 2010 - matches responses from a postenumeration survey to the census to evaluate coverage. While the DA estimates showed the 4.6 percent net undercount for young children, the CCM results only found a 0.7 percent net undercount [5]. The DA estimate of net undercount for young children is preferred over the CCM estimate because the DA estimates for this cohort were developed using primarily birth records, which are believed to be near complete in the U.S. The CCM estimate comes from a survey, and there may be correlation bias between the survey and the census [6], which can lead to an underestimation of the total population by the DSE approach.

There is a growing literature on the coverage of children in the decennial census [1,3,6-8]; however, little is known about the coverage of children in demographic surveys. Prior research has found that households with children tend to have lower levels of refusal and non-contact for surveys, regardless of the number or age of children $[9,10]$. The increased cooperation for households with children may be a simple function of caregivers being at home during the day and having more time to participate in a survey or, as some have hypothesized, households with children may have higher levels of social integration and social obligation [10].

There are important differences between the design of household surveys and censuses that may affect coverage. First, surveys are a sample of the population while the census is a complete enumeration. If there are errors in the sampling frame, then some groups may not be fully represented in the sample. Demographic surveys often use complex sampling designs to ensure a representative sample, but even then, there may be some hard-to-survey populations that are not fully represented.

In addition, the nonresponse followup operations for household surveys are not as extensive as they are for the census. While some surveys use multiple modes of data collection to improve response rates, the modes that resemble the field nonresponse followup operation from the census may only be used for a sample of the total households that do not return a mail questionnaire [11]. Furthermore, demographic surveys do not use proxy responses from non-household members to obtain information about households that do not respond resulting in lower response rates. Panel attrition from longitudinal surveys may lower coverage rates, which is not an issue for the decennial census.

Finally, surveys do not have the same marketing and information campaigns as the census. The paid advertising budget for the 2010 Census was $\$ 167$ million and one of the expressed goals of the program was to "reduce the differential undercount" [12]. In fact, the marketing campaign for the 2010 Census included a specific project encouraging parents to include young children on their census form. The "Children Count Too" ad was a short cartoon featuring Dora the Explorer from a popular children's television show talking about the importance of including infants and young children on the census forms. 
While survey design issues may affect coverage in general, there may be other aspects of the survey and census that cause coverage error specifically for young children. Error in rostering household members may affect young children disproportionately [4,13]. Most demographic surveys ask respondents to roster household members based on usual or current residence. However, complex living arrangements, irregular housing, mobility, concealment and distrust, language barriers, and interviewer error may also cause ambiguity about residence and cause coverage error, as shown in ethnographic evaluations of decennial censuses [14-17]. Research has also shown that divorced parents have difficulty describing the living arrangements of children when the child spends substantial time in both parents' homes [18]. Other research has found that when responding to the Fragile Families and Child Wellbeing study, unmarried parents often differed when identifying the resident parent for the child [19].

Young children may also be part of subfamilies that are omitted from the household roster. Another project related to the coverage of children in the census focused on the coverage of young mothers in the ACS. This research found that recent unmarried mothers unmarried women who had given birth in the past 12 months - age 15 to 19 and 20 to 24 had higher levels of estimated net undercount than other age groups, 30.9 and 13.7 percent, respectively [20]. Although females age 15 to 19 and 20 to 24 do not have net undercounts in the census, young mothers may be undercounted, but are part of the college-aged cohort that tends to be over-counted in the census.

\subsection{Research questions}

This paper answers the following research questions.

1. Do coverage rates in demographic surveys vary by age? If so, to what extent are young children undercovered compared to other age groups? ${ }^{1}$

2. How do the coverage rates of young children vary by race and Hispanic origin?

3. How are coverage patterns in demographic surveys similar to coverage in the census?

\footnotetext{
${ }^{1}$ In this analysis, we use the terms undercovered and overcovered to interpret the coverage rates from the surveys. For more information about coverage error in surveys, see Chapter 5 in United States Federal Committee on Statistical Methodology [21].
}

\section{Data and methods}

In this report, we analyze coverage rates by age, race, and Hispanic origin for three major demographic surveys conducted by the Census Bureau: the ACS, CPS and SIPP. These surveys are each controlled to the annual population estimates that are also produced by the Census Bureau. We use the same methodology to estimate coverage rates that the different survey areas use to produce official estimates of coverage [22]. In this section, we discuss the method for measuring coverage in the surveys and then provide a description of the population estimates and the surveys used in the analysis.

\subsection{Measuring coverage in surveys}

Coverage rates are a measure of coverage error in a survey and are calculated as the ratio of the population estimate from the survey to an independent population estimate. Coverage error is typically the result of errors in the sampling frame that cause the target population not to be fully covered by the survey. Whether coverage error leads to coverage bias in the survey statistics depends on 1) the proportion of the target population that is not covered by the frame and 2) how different the characteristics of the uncovered population are from the covered population [23,24].

The coverage rate reports the proportion of the frame that is covered by the survey. The percent undercount of a population can be expressed as the difference between the coverage rate and full coverage (1.00) multiplied by 100 . For example, if a population has a coverage rate of 0.90 in a survey, the percent undercount of that population would be $(1.00-0.90) \times 100$, or 10 percent. To measure coverage bias on survey statistics, we would need to know the characteristics of this 10 percent of the population and how they are different from the 90 percent that was covered by the survey. Because we do not have information on the characteristics of the population that was not covered by the survey, we focus the analysis on the proportion of the target population that is not covered by the survey. In short, we are measuring coverage error in the survey and not coverage bias in the survey estimates.

The Census Bureau's Master Address File (MAF), which is a permanent list of addresses in the United States, is used as the sampling frame for the ACS, CPS, and SIPP. The MAF is considered an exceptionally complete frame for drawing a sample, but it still has some limitations because of the frequency at which 
the file is updated, difficulties recording new housing construction, issues with multi-unit addresses, and housing in rural areas [25]. However, it is unclear how these limitations would produce differential coverage by age or an undercount for young children specifically. Therefore, the undercount of young children in demographic surveys could also result from other types of survey error, in addition to frame errors.

For example, the coverage rates for young children could also be low if households with young children are not responding to the survey (nonresponse error). In addition, if children are left off household rosters or if age is misreported then the coverage rate for young children will be low (measurement error). In this report, we interpret the undercount, or the difference between the coverage rate and full coverage (1.00), to result from a combination of frame, nonresponse, and measurement errors. While sampling error could also be an issue, we account for this by estimating the sample variance of the coverage rates and reporting margins of error at the 90-percent confidence interval with the results.

\subsection{Cumulative coverage errors}

The population estimates used to calculate the coverage rates are based on the most recent decennial census. Therefore, coverage errors in the census will be reproduced in the population estimates and will upwardly bias the coverage rates from the surveys [26]. We addressed this issue in our analysis in two ways. First, we use survey data from 2009 and 2015 where the population estimates of young children are not based on the census because these cohorts were born after the census. This applies to the 0 to 4 and 5 to 9 age groups in the 2009 data and the 0 to 4 age group in the 2015 files. However, for the older age groups, there will still be the potential for "cumulative" coverage error because the coverage rate in the survey may be under- or overestimated because of coverage errors in the census.

The second approach to address this issue is to calculate the cumulative coverage errors directly by adjusting the population estimate for estimated coverage errors in the census base. We adjust the population estimate by calculating the change to the cohort since the previous census and then adding that change to the DA estimate for the cohort. The DA estimate represents an independent population estimate of the cohort at the time of the census that is not based on the previous census or the census being evaluated.
For example, to calculate the adjusted coverage rate for the 5 to 9 population in a 2015 survey, we would use the following equations:

$$
\begin{aligned}
& \text { Adjusted coverage rate } \\
& =\frac{2015 \text { Survey estimate } 5 \text { to } 9}{2015 \text { Adjusted Population estimate } 5 \text { to } 9} \\
& 2015 \text { Survey estimate } 5 \text { to } 9 / \\
& 2015 \text { Population estimtate } 5 \text { to } 9-2010 \\
& \text { Census } 0 \text { to } 4+2010 \text { DA estimate } 0 \text { to } 4 \\
& =\frac{2015 \text { Survey estimate } 5 \text { to } 9}{2010 \text { DA estimate } 0 \text { to } 4+\triangle}
\end{aligned}
$$

Here, $\triangle$ is the change in the cohort between the 2010 Census and the Vintage 2015 population estimates. We add the $\triangle$ to the estimate of the population age 0 to 4 from the 2010 DA to produce the 2015 adjusted coverage estimate of the population age 5 to 9 . For this analysis, we use data from the 2000 DA, Census 2000, and Vintage 2009 population estimates to adjust the coverage rates in the 2009 surveys and data from the 2010 DA, 2010 Census, and Vintage 2015 population estimates to adjust the coverage rates in the 2015 surveys.

This method produces an approximation of the true cohort size; however, coverage in the census can still affect the estimates of population change since the previous census. For instance, some of the estimates processing uses rates and proportions that are applied to the census base population. The estimates of cohort change after the census would be biased downward if the rates and portions were applied to populations that were undercounted in the previous census. While we do not have a method for addressing this, the rates and proportions are used to estimate the number of deaths to the cohort, which are relatively low for the age groups that we focus on in the analysis. ${ }^{2}$

\subsection{Population Estimates}

The Population Estimates use the most recent decennial census counts as the base population and a components of population change model (births, deaths, and migration) to produce annual estimates of the U.S. population. The estimates are produced for the nation, states, and counties by age, sex, race, and Hispanic

\footnotetext{
${ }^{2}$ Rates and proportions are also used to estimate domestic migration at the state and county levels. For this analysis, we use nationallevel population estimates.
} 
origin. In addition, the Census Bureau produces subcounty estimates of total population and housing units, which are also used as controls for some surveys (e.g., the ACS).

An assumption of the method used to measure coverage in the demographic surveys is that the differences between the survey estimates and the population estimates indicate error only in the survey. However, there may also be errors in the population estimates. For example, the national birth estimates are produced using vital records from the National Center for Health Statistics (NCHS). The birth data with full demographic and geographic detail are not current when the population estimates are produced and are generally lagged two years. The Population Estimates Program also receives the national total for births for the year prior to the vintage year, which is used to control the most recent full data. The estimates for the vintage year are projected using fertility rates and an estimated at risk population. For the Vintage 2009 population estimates, the most recent full year of data was 2007. The 2008 birth estimates were produced using the 2007 data but controlled to the national total of births for 2008. The 2009 birth estimates were developed using the projection method described above.

As long as the trends in births are consistent during the years without full data, the projected estimates for these cohorts should be relatively accurate. However, there was a decline in birth rates during the Great Recession from 2008 to 2013, which may cause the birth components for 2009 to be overestimated [27]. To test the impact of this on the analysis, we compared the projected births in the Vintage 2009 estimates for 2009 to the actual births the Vintage 2010 estimates for $2009 .{ }^{3}$ We found that the projected births in the Vintage 2009 population estimates were 1.6 percent higher than the actual birth data for 2009 in the Vintage 2010 population estimates. Although this error in the projected births would bias the population estimates for young children upward, this would only have a small affect the coverage rates for this population.

There could also be error in the estimates of births by race and Hispanic origin in the Vintage 2009 births for 2008. Because the birth component for 2008 was controlled to a national total, the estimates may not reflect new trends in birth rates for a specific race or

\footnotetext{
${ }^{3}$ Each vintage of population estimates includes a time series of the estimates from vintage year back to the previous census. Therefore, the Vintage 2010 population estimates will include an estimate of births for 2009 .
}

Hispanic origin group. This would potentially bias the coverage rates for young children of a particular race and Hispanic origin group if the increase or decline in birth rates for this group was different than the change for the total population. The birth records used to produce the Vintage 2015 estimates would have included recession era trends and yet we do not see large differences between the coverage rates for young children in 2009 and 2015.

Another source of possible error in the population estimates comes from uncertainty in estimating migration. International and domestic migration are the most difficult components of population change to accurately estimate. The estimates of international migration for young children are low relative to births, but they have more impact on the older ages, which we also examine in the results. Evaluations of the recent estimates of net international migration have found that the estimates may be too low, which would bias the coverage rates downward [28].

In addition, there may be classification error for the race and Hispanic origin groups between the demographic surveys and the population estimates. The 2000 and 2010 censuses allowed respondents to select Some Other Race (SOR) as their race category. However, the population estimates uses a modified race method that collapses SOR into the other race categories [29]. The modified race data have 31 categories, which include the five standard single race groups from the Office of Management and Budget (White, Black, American Indian/Alaska Native, Asian, and Native Hawaiian/Pacific Islander) and all possible combinations of the single race groups. While the demographic surveys allow respondents to report SOR, the survey estimates are not controlled to estimates of the SOR population, but to larger or aggregated race categories. Still, the differences in how the SOR category is handled in the population estimates and the surveys may potentially bias downward the coverage rates for the non-Hispanic Black alone population, making the differential between it and the non-Hispanic White population seem larger than it actually is.

There are several examples of misclassification in race between the census and population estimates and demographic surveys. Studies have shown discrepancies between race reporting in the Census 2000 data (the base for the Vintage 2009 estimates) and the Census 2000 Supplementary Survey, which was an expansion of ACS prior to full implementation [30,31]. Other research has found discrepancies in race reporting between the 2010 Census and the ACS, but mainly for 
the American Indian Alaska Native (AIAN) population [32]. There is also evidence of classification error between the Census 2000 data and the CPS [33].

\subsection{American Community Survey}

The ACS is a multi-mode survey conducted throughout the year with an initial sample size of approximately 3 million housing units. The ACS sample includes both housing units and group quarters facilities. In this report, we focus on the population living in housing units and exclude the group quarters data. The ACS collects the demographic, economic, and social data that were previously collected through the longform of the decennial census as well as additional questions. ACS estimates are produced for 1-year and 5year periods. For this analysis, we use data from the 2009 and 2015 1-year ACS files.

The Census Bureau uses a ratio estimation procedure to control the ACS estimates to the housing-unit and population estimates discussed above. Prior to the ratio estimation, there are several intermediate steps in the weighting process including an adjustment for the probability of selection into the sample (base weight), noninterview bias, variation in monthly sampling, and mode bias. The ACS estimates that we use to calculate coverage rates include all of these adjustments, but not housing-unit or population controls. We refer to these as the "uncontrolled" ACS estimates. The coverage rates for ACS estimates by specific demographic group Y (e.g., Hispanics age 0 to 4 ) can be estimated using Eq. (3):

$$
\begin{aligned}
& \text { ACS coverage rate of group } Y \\
& =\frac{\text { uncontrolled ACS estimate of group } Y}{\text { Population Estimate of group } Y}
\end{aligned}
$$

The uncontrolled ACS estimates of group Y use data weighted with all of the survey weights prior to the housing-unit and population controls.

\subsection{Current Population Survey}

The CPS is a monthly telephone survey of approximately 60,000 households in the United States. The primary purpose for the CPS is to collect data on employment, but the survey also includes information on the demographic, economic, and social characteristics of respondents. The universe for this survey is the civilian non-institutional population. For this analysis, we use the 2009 and 2015 CPS Annual Social and Economic (ASEC) Supplement.
The sample size for the ASEC $(99,000)$ is considerably larger than the base CPS sample because the ASEC includes several additional subsamples. The first is a sample of Hispanic households, which increases the sample by 4,500 households. The second is the Children's Health Insurance Program (CHIP) sample which expands the sample by 34,500 households and provides information about CHIP. The CHIP sample includes 1) one-quarter of the February and April CPS sample, 2) selected households from the preceding November sample, and 3) an increased sample in states with high sampling errors for uninsured children [34].

Similar to the ACS, the Census Bureau uses a ratio adjustment procedure to control the CPS data to the Population Estimates. Before the CPS data are controlled to population estimates, they are weighted to account for the probability of selection into the sample, special weighting adjustments, and noninterview adjustment. The first stage of the ratio adjustment accounts for the variance in the state-level estimates caused by the sampling of primary sampling units (PSU), or the between-PSU variance [34]. PSUs are made up of a metropolitan area, large county, or a cluster of smaller counties. The PSUs are grouped into homogeneous strata based on data from the decennial census and other sources and then used to develop the CPS sample. In the second-stage ratio adjustment, the data are controlled to the Population Estimates. Therefore, we use the CPS estimates after the first-stage ratio adjustment as the "uncontrolled" estimate in calculating coverage rates. We divide the first-stage ratio adjusted estimates by age, race, and Hispanic origin by the Population Estimate for the corresponding group. The coverage rates for CPS estimates by specific demographic group Y can be estimated using Eq. (4):

$$
\begin{aligned}
& \text { CPS coverage rate for group } Y \\
& =\frac{\text { first stage ratio adjustment for group } Y^{(4)}}{\text { Population Estimate of group } Y}
\end{aligned}
$$

The first stage ratio adjustment is the stage in the weighting process just prior to controlling to the population estimates.

\subsection{Survey of Income and Program Participation}

The SIPP is a nationally representative panel survey of the civilian non-institutionalized population that follows households over a specific period. The SIPP includes a variety of demographic, social, and economic indicators, but the main purpose of the survey is to 
measure income and program participation. The interviews are conducted during a personal visit or decentralized phone call. Each member of the household 15 years of age and older is personally interviewed and proxy responses are only used for children younger than 15 and household members that are unavailable at the time of the interview. For the 2008 Panel, households were interviewed every four months over a fiveyear period beginning in September 2008 [35]. The initial wave of the 2008 Panel included approximately 52,000 households. For this analysis, we used data from Waves 2 and 3 of the 2008 Panel, which were collected in 2009 and correspond roughly to the same collection months as the CPS ASEC. ${ }^{4}$ The 2015 data, which would be part of the 2014 Panel, were not available at the time of publication.

The weights in the SIPP files can be used to develop samples based on month, year, or panel. Within each type of weight there are several possible units of analysis including person, household, family, and related subfamily. The basic process for constructing the weights is the same for each type of weight. First, there is a base weight that represents the probability of selection into the sample. Next, there is an adjustment for subsampling within the cluster. After Wave 1, there is an adjustment for movers. The next stage in the weighting process is a nonresponse adjustment that accounts for sample nonresponse and attrition. Finally, there is a post-stratification (second-stage calibration) adjustment where the survey estimates are controlled to the official population estimates [36].

To calculate coverage rates for the SIPP data, we use the survey estimate weighted using nonresponse adjustment. The nonresponse adjustment is the last stage in the weighting process before the data are controlled to the population estimates. The coverage rates for SIPP estimates for a specific demographic group Y can be estimated using Eq. (5):

$$
\begin{aligned}
& \text { SIPP coverage rate for group } Y \\
& =\frac{\text { nonresponse adjustment of group } Y}{\text { Population Estimate of group } Y}
\end{aligned}
$$

\section{Results}

We calculated coverage rates by age, race, and Hispanic origin for the ACS, CPS, and SIPP. Coverage

\footnotetext{
${ }^{4}$ Wave 1 was excluded because the data were collected in the fall of 2008 .
}

rates can only be calculated by the same demographic characteristics as the Official Population Estimates. Therefore, we are not able to produce coverage rates by other social or economic characteristics such as household structure, poverty status, or income. We did calculate coverage rates by sex for these different groups, but did not find significant difference between males and females under 18 years of age. While there can be significant sex differentials in coverage, these tend to be for adults (e.g. Black males) and the focus of this report is on the coverage of young children. Therefore, we do not report sex in the results.

\subsection{American Community Survey}

The coverage rates by age for the 2009 and 2015 single-year ACS are presented in Table 1. This table reports both the coverage rate and the adjusted coverage rate with their associated margins of error. The adjusted coverage rate accounts for coverage in the census and provides an estimate of the cumulative coverage error of the census base and the survey. In 2009, the coverage rate for the population aged 0 to 4 was 0.89 . The population aged 5 to 9 had a coverage rate of 0.94 . Because these populations were born after the 2000 Census, their adjusted coverage rates do not change. For the populations age 10 to 17 and 18 years and older, the adjusted coverage rates were 0.96 and 0.95 , respectively. The coverage rate for young children implies an 11 percent undercount for this population in the 2009 ACS, which was considerably higher than the undercount for the other three age groups.

In 2015 , the coverage rate for young children was 0.87, which implies a 13 percent undercount (Table 1). The adjusted coverage rate for this population does not change because this cohort was born after the census. The coverage rate for the population age 5 to 9 was 0.94 and the adjusted coverage rate was 0.90 . The adjusted coverage rate for this population reflects the high undercount for this cohort in the 2010 Census, which was between the ages of 0 and 4 in 2010. The adjusted undercount for the 10 to 17 and 18 years and older populations were 0.92 and 0.93 , respectively. Even after adjusting the ACS coverage rates for the coverage error in the census base, young children have a higher undercount rate in the survey than any other age group in both of the years examined here.

The undercount for young children in the ACS also varies by race and Hispanic origin. Table 2 reports the coverage rates and margins of error by age, race, and Hispanic origin for the 2009 and 2015 single-year ACS 
Table 1

American Community Survey adjusted coverage rates by age: 2009 and 2015

\begin{tabular}{|c|c|c|c|c|c|c|c|c|}
\hline \multirow[t]{2}{*}{ Age } & \multicolumn{4}{|c|}{2009} & \multicolumn{4}{|c|}{2015} \\
\hline & Coverage rate & $\mathrm{MOE}$ & Adjusted coverage rate & $\mathrm{MOE}$ & Coverage rate & $\mathrm{MOE}$ & Adjusted coverage rate & $\mathrm{MOE}$ \\
\hline 0 to 4 & 0.89 & 0.004 & 0.89 & 0.004 & 0.87 & 0.004 & 0.87 & 0.004 \\
\hline 5 to 9 & 0.94 & 0.005 & 0.94 & 0.005 & 0.94 & 0.004 & 0.90 & 0.004 \\
\hline 10 to 17 & 0.99 & 0.004 & 0.96 & 0.004 & 0.94 & 0.004 & 0.92 & 0.004 \\
\hline $18+$ & 0.95 & 0.002 & 0.95 & 0.002 & 0.92 & 0.002 & 0.93 & 0.002 \\
\hline
\end{tabular}

Note: The margin of error (MOE) was calculated using the 90-percent confidence interval. Source: 2009 and 2015 American Community Survey files, 2000 and 2010 Census, 2000 and 2010 Demographic Analysis estimates.

Table 2

American Community Survey coverage rates by age, race, and Hispanic origin: 2009 and 2015

\begin{tabular}{|c|c|c|c|c|c|c|c|c|}
\hline \multirow[t]{2}{*}{ Age } & \multicolumn{2}{|l|}{ Total } & \multicolumn{2}{|c|}{ Non-Hispanic White alone } & \multicolumn{2}{|c|}{ Non-Hispanic Black alone } & \multicolumn{2}{|c|}{ Hispanic } \\
\hline & Coverage rate & MOE & Coverage rate & MOE & Coverage rate & MOE & Coverage rate & MOE \\
\hline \multicolumn{9}{|l|}{2009} \\
\hline $0-4$ & 0.89 & 0.004 & 0.90 & 0.005 & 0.86 & 0.012 & 0.85 & 0.009 \\
\hline $5-9$ & 0.94 & 0.005 & 0.94 & 0.005 & 0.92 & 0.012 & 0.93 & 0.009 \\
\hline $10-17$ & 0.99 & 0.004 & 0.97 & 0.005 & 0.96 & 0.012 & 1.04 & 0.010 \\
\hline $18+$ & 0.95 & 0.002 & 0.96 & 0.003 & 0.89 & 0.004 & 0.92 & 0.005 \\
\hline \multicolumn{9}{|l|}{2015} \\
\hline $0-4$ & 0.87 & 0.004 & 0.91 & 0.006 & 0.79 & 0.012 & 0.82 & 0.009 \\
\hline $5-9$ & 0.94 & 0.004 & 0.97 & 0.006 & 0.87 & 0.013 & 0.93 & 0.008 \\
\hline $10-17$ & 0.94 & 0.004 & 0.95 & 0.004 & 0.88 & 0.011 & 0.94 & 0.008 \\
\hline $18+$ & 0.92 & 0.002 & 0.95 & 0.003 & 0.85 & 0.004 & 0.87 & 0.004 \\
\hline
\end{tabular}

Note: The margin of error (MOE) was calculated using the 90-percent confidence interval. Source: 2009 single-year American Community Survey and Vintage 2009 Population Estimates.

data. In this part of the analysis, we do not report an adjusted coverage rate because the data are not available to make such an adjustment by the specific race and Hispanic origin groups shown in the table and the focus here is on the differentials in the coverage rate by race and Hispanic origin by age. In 2009, the highest coverage rate for young children was 0.90 for the non-Hispanic White alone population. The coverage rate for young non-Hispanic Black alone and Hispanic children were 0.86 and 0.85 , respectively. The coverage rates indicate a 14 percent undercount for nonHispanic Black alone children and 15 percent undercount for Hispanic children in the 2009 ACS. The coverage rates for the non-Hispanic Black alone and Hispanic populations were not statistically different.

We find similar patterns of differential coverage by race and Hispanic origin in the 2015 ACS (Table 2). In that year, the highest coverage rate among young children was 0.91 for the non-Hispanic White alone population. The coverage rate for non-Hispanic Black alone population age 0 to 4 was 0.79 , indicating an undercount of 21 percent in the survey. Young Hispanic children had a coverage rate of 0.82 , which implies an undercount of 18 percent.

The other age groups also showed differential coverage in the ACS by race and Hispanic origin (Table 2).
The non-Hispanic Black alone population age 18 and older was undercovered by 11 percent in the 2009 ACS and 15 percent in the 2015 ACS. This was significantly higher than the undercount for the non-Hispanic White alone population, which had a 4 percent undercount in 2009 and 6 percent undercount in 2015. This is consistent with coverage patterns in the decennial census where the Black population has historically had larger undercounts than the White population [37].

Hispanics age 10 to 17 actually had a coverage rate of 1.04 , meaning that this population was over-counted in the 2009 ACS (Table 2). This is also consistent with the 2010 DA Hispanic estimates that found a significant overcount for Hispanic teenagers in the 2010 Census [38]. The significantly lower coverage rates for the non-Hispanic Black and Hispanic populations compared to the non-Hispanic white alone population may indicate true differences in survey coverage or could also reflect the misclassification of race and Hispanic origin between the population estimates and the ACS.

Technically, the coverage rates presented in Tables 1 and 2 include both housing unit coverage error and within-household coverage error. To understand which of these two types of coverage error is contributing the most to the total coverage rates, we decomposed the total coverage rate into housing unit and within- 
Table 3

Decomposition of the total coverage rate by housing unit and within-household coverage: 2009 and 2015

\begin{tabular}{|c|c|c|c|c|c|c|}
\hline \multirow[t]{2}{*}{ Age } & \multicolumn{3}{|c|}{2009} & \multicolumn{3}{|c|}{2015} \\
\hline & Total & Housing unit coverage & Within-household coverage & Total & Housing unit coverage & Within-household coverage \\
\hline $0-4$ & 0.89 & 0.99 & 0.90 & 0.88 & 0.99 & 0.89 \\
\hline $5-9$ & 0.96 & 0.99 & 0.97 & 0.94 & 0.99 & 0.95 \\
\hline $10-17$ & 0.97 & 0.99 & 0.98 & 0.94 & 0.99 & 0.95 \\
\hline $18+$ & 0.95 & 0.99 & 0.95 & 0.92 & 0.99 & 0.94 \\
\hline
\end{tabular}

Note: Total coverage may not equal the Total reported in Tables 1 and 2. Source: 2009 and 2015 single-year American Community Survey.

Table 4

Current Population Survey adjusted coverage rates by age: 2009 and 2015

\begin{tabular}{|c|c|c|c|c|c|c|c|c|}
\hline \multirow[t]{2}{*}{ Age } & \multicolumn{4}{|c|}{2009} & \multicolumn{4}{|c|}{2015} \\
\hline & Coverage rate & MOE & Adjusted coverage rate & MOE & Coverage rate & MOE & Adjusted coverage rate & $\mathrm{MOE}$ \\
\hline 0 to 4 & 0.82 & 0.020 & 0.82 & 0.020 & 0.82 & 0.029 & 0.82 & 0.029 \\
\hline 5 to 9 & 0.90 & 0.020 & 0.90 & 0.020 & 0.89 & 0.028 & 0.85 & 0.027 \\
\hline 10 to 17 & 0.92 & 0.018 & 0.89 & 0.017 & 0.89 & 0.029 & 0.88 & 0.028 \\
\hline $18+$ & 0.88 & 0.006 & 0.88 & 0.006 & 0.87 & 0.019 & 0.88 & 0.019 \\
\hline
\end{tabular}

Note: The margin of error (MOE) was calculated using the 90-percent confidence interval. Source: March 2015 Base Current Population Survey.

household coverage by age for the 2009 and 2015 single-year ACS files. The ACS data are controlled to both housing unit and population controls that are developed by the Population Estimates Program. The coverage rates presented in Tables 1 and 2 were calculated using the survey estimates before either the housing unit or population controls and the independent population estimates were applied. By using the weighted estimates at each stage of this process - prehousing unit control, pre-population control, and final person weight - we can decompose the coverage rate into housing unit coverage and within-household coverage [32]. Factoring the coverage rates into these two parts allows us to measure the extent to which the total coverage error was due to within-household coverage error and housing unit coverage error [32].

Table 3 reports the decomposition of the total coverage rate into housing unit and within-household (population) coverage error. The total coverage rate in Table 3 may differ slightly from what was reported in Tables 1 and 2 because of minor differences in the method used to calculate the separate types of coverage error.

The total coverage rate is the product of the housing unit and population coverage rates. For this table, we do not adjust the within household coverage rates for coverage error in the census or the housing unit coverage rates for errors in the MAF, even though there is some evidence of coverage error in the ACS sampling frame [32]. In general, the housing unit coverage rates in the ACS data are quite high, indicating that the survey frame and the census frame are very similar. In addition, the results show that nearly all of the total coverage error comes from within-household coverage [22].
In addition, we do not find variation in the housing unit coverage by age.

\subsection{Current Population Survey}

Table 4 reports the coverage rates and adjusted coverage rates by age for the 2009 and 2015 CPS ASEC files. The adjusted coverage rate accounts for coverage for the cohort in the census base of the population estimates. In general, we find that the coverage rates in the CPS tend to be lower than the ACS coverage rate, which is consistent with other studies [32]. These differences in coverage rates between the ACS and CPS could be a function of lower response rates and greater measurement errors in the CPS. Nonetheless, the CPS coverage rates follow similar patterns by age to the ACS coverage rates.

In 2009 , the coverage rate for young children was 0.82 , which indicates that this population was undercovered in the CPS by 18 percent. The coverage rate for young children was significantly lower than coverage rate for the other age groups (Table 4). The population aged 5 to 9 and the 10 to 17 were undercovered by 10 percent and 11 percent, respectively. The population age 18 and older had a coverage rate of 0.88 . In the 2015 CPS, young children were undercovered by 18 percent. The population age 5 to 9 had a coverage rate of 0.89 but an adjusted coverage rate of 0.85 . The adjusted coverage rate accounts for the coverage of this cohort in the 2010 Census, which was undercovered by 4.6 percent. After accounting for cumulative coverage error, the coverage rate for children age 0 to 4 was not 
Table 5

Current Population Survey coverage rates by age, race, and Hispanic origin: 2009 and 2015

\begin{tabular}{|c|c|c|c|c|c|c|c|c|}
\hline \multirow[t]{2}{*}{ Age } & \multicolumn{2}{|l|}{ Total } & \multicolumn{2}{|c|}{ Non-Hispanic White alone } & \multicolumn{2}{|c|}{ Non-Hispanic Black alone } & \multicolumn{2}{|c|}{ Hispanic } \\
\hline & Coverage rate & MOE & Coverage rate & MOE & Coverage rate & $\mathrm{MOE}$ & Coverage rate & MOE \\
\hline & & & & 2009 & & & & \\
\hline 0 to 4 & 0.82 & 0.02 & 0.85 & 0.03 & 0.71 & 0.06 & 0.79 & 0.05 \\
\hline 5 to 9 & 0.90 & 0.02 & 0.92 & 0.03 & 0.83 & 0.05 & 0.92 & 0.05 \\
\hline 10 to 17 & 0.92 & 0.02 & 0.92 & 0.02 & 0.86 & 0.05 & 0.94 & 0.05 \\
\hline $18+$ & 0.88 & 0.01 & 0.90 & $\begin{array}{l}0.01 \\
2015\end{array}$ & 0.80 & 0.02 & 0.85 & 0.03 \\
\hline 0 to 4 & 0.82 & 0.03 & 0.89 & 0.04 & 0.71 & 0.06 & 0.77 & 0.05 \\
\hline 5 to 9 & 0.89 & 0.03 & 0.95 & 0.04 & 0.73 & 0.06 & 0.85 & 0.05 \\
\hline 10 to 17 & 0.89 & 0.03 & 0.92 & 0.04 & 0.81 & 0.05 & 0.88 & 0.04 \\
\hline $18+$ & 0.87 & 0.02 & 0.91 & 0.02 & 0.78 & 0.04 & 0.81 & 0.03 \\
\hline
\end{tabular}

Note: The margin of error (MOE) was calculated using the 90-percent confidence interval. Source: March 2009 and 2015 Base Current Population Survey.

statistically different from the adjusted coverage rate for the population aged 5 to 9 .

We also compared the CPS coverage races by race and Hispanic origin (Table 5). Similar to the ACS tables, we produced coverage rates for the total, nonHispanic White alone, non-Hispanic Black alone, and Hispanic populations by age. Again, we do not show an adjusted coverage rate by race and Hispanic origin because the data to make that adjustment are not available for these categories. In 2009, the estimated undercount for the non-Hispanic White alone population aged 0 to 4 was 15 percent. The estimated undercounts for the non-Hispanic Black alone and Hispanic populations aged 0 to 4 were 29 percent and 21 percent, respectively.

The 2015 CPS also shows differential coverage by race and Hispanic origin (Table 5). In that year, the coverage rate for the non-Hispanic White alone population was 0.89 compared to 0.71 for the non-Hispanic Black alone population and 0.77 for Hispanics. These coverage rates imply that the non-Hispanic Black alone population aged 0 to 4 had a 29 percent undercount and the Hispanic population aged 0 to 4 had a 23 percent undercount in the 2015 CPS ASEC. Again, the differential coverage rates may be measuring differences in coverage across race and Hispanic origin groups, but could also reflect differences in classification of race and origin between the survey and the population estimates.

\subsection{Survey of Income and Program Participation}

We analyzed the coverage rates and adjusted coverage rates by age for Waves 2 and 3 of the 2008 Panel of the SIPP (Table 6). The collection period for these data corresponds with the collection period for the 2009 CPS ASEC. We do not show the 2015 SIPP data be-
Table 6

Survey of Income and Program Participation adjusted coverage rates by age: 2009

\begin{tabular}{lcccc}
\hline Age & Coverage rate & MOE & Adjusted coverage rate & MOE \\
\hline 0 to 4 & 0.81 & 0.020 & 0.81 & 0.020 \\
5 to 9 & 0.88 & 0.024 & 0.88 & 0.024 \\
10 to 17 & 0.88 & 0.019 & 0.86 & 0.019 \\
$18+$ & 0.87 & 0.007 & 0.87 & 0.007 \\
\hline
\end{tabular}

Note: The margin of error (MOE) was calculated using the 90percent confidence interval. Source: Survey of Income and Program Participation, 2008 (Waves 2 and 3) Panel.

cause they were not available at the time of this writing. The coverage rates for the SIPP were lower than the estimated coverage rates for the ACS, which was similar to the CPS. This may reflect the panel design of the SIPP and attrition between waves or that the SIPP questionnaire is considerably longer than the instruments for the ACS that results in lower response rates and possibly greater measurement errors.

The coverage rate for young children was 0.81 , indicating an estimated undercount of 19 percent, which was lower than the coverage rates for the other age groups. The population aged 5 to 9 had a coverage rate of 0.88 while the population 10 to 17 had a coverage rate of 0.88 and an adjusted coverage rate of 0.86 . That the adjusted coverage rate was lower than the initial coverage rate for this age group implies cumulative coverage error between the survey and the Census 2000 counts. The adjusted coverage rate for the population age 18 years and older was 0.87 , which did not change when we adjusted the population estimate for coverage error in the census.

As with the ACS and CPS data, there was variation in coverage rates by race and Hispanic origin. Similar to the other surveys, we report coverage rates for the non-Hispanic White alone, non-Hispanic Black alone, and Hispanic populations (Table 7). For the non- 
Table 7

Survey of Income and Program Participation coverage rates by age, race, and Hispanic origin: 2009

\begin{tabular}{|c|c|c|c|c|c|c|c|c|}
\hline \multirow[t]{2}{*}{ Age } & \multicolumn{2}{|l|}{ Total } & \multicolumn{2}{|c|}{ Non-Hispanic White alone } & \multicolumn{2}{|c|}{ Non-Hispanic Black alone } & \multicolumn{2}{|c|}{ Hispanic } \\
\hline & Coverage rate & MOE & Coverage rate & MOE & Coverage rate & MOE & Coverage rate & MOE \\
\hline 0 to 4 & 0.81 & 0.02 & 0.84 & 0.03 & 0.73 & 0.05 & 0.72 & 0.04 \\
\hline 5 to 9 & 0.88 & 0.02 & 0.89 & 0.03 & 0.90 & 0.07 & 0.79 & 0.05 \\
\hline 10 to 17 & 0.88 & 0.02 & 0.90 & 0.02 & 0.84 & 0.05 & 0.81 & 0.04 \\
\hline $18+$ & 0.87 & 0.01 & 0.90 & 0.01 & 0.85 & 0.03 & 0.68 & 0.02 \\
\hline
\end{tabular}

Note: The margin of error (MOE) was calculated using the 90-percent confidence interval. Source: Survey of Income and Program Participation, 2008 (Waves 2 and 3) Panel.

Hispanic White population, the estimated undercount for young children was 16 percent (Table 7). The estimated undercount for the non-Hispanic Black alone population aged 0 to 4 was 27 percent. We estimate that the Hispanic population aged 0 to 4 was undercovered by 28 percent in the SIPP.

The Hispanic population aged 18 and older had an adjusted coverage rate of 0.68 , implying an undercount of 32 percent. However, we did not adjust this estimate for coverage error in the census. The Hispanic population is the only group where the undercount for young children was not higher than one of the other age groups. Again, this was the only instance - within all three surveys - where the coverage rate for young children was not lower than another age group within the same race or ethnic category. That the coverage rate for this group was so low could indicate that the population estimates used to control the survey overestimated Hispanics. However, we do not find the same pattern in the ACS or CPS data. There could also be differences in classification between the SIPP and the population estimates, but this is less likely with Hispanic origin than race. Finally, there could be some reason why the initial rostering rules for the SIPP surveys have a differential impact on Hispanics, but to include this in the analysis is beyond the scope of the current paper.

\section{Conclusion}

This paper focuses on the coverage of young children age 0 to 4 in demographic surveys. While there is a growing literature on the coverage of young children in the decennial census, less is known about the coverage of this population in surveys. Previous ACS coverage studies by age used the 2010 Census as a benchmark and did not detect the errors that become obvious when we used the population estimates as the benchmark [22]. We found that across the three largest demographic surveys conducted by the Census Bureau ACS, CPS, and SIPP - young children tend to have lower coverage rates than other age groups. We also found that coverage rates for young children in the ACS vary by race and Hispanic origin. However, the difference in coverage rates for young children in the CPS and SIPP by race and Hispanic origin were not statistically significant.

The patterns of coverage by age, race, and Hispanic origin in the demographic surveys are very similar to patterns of net undercount in the decennial census, as measured by Demographic Analysis. Specifically, young children have higher undercounts than older age groups, and generally non-Hispanic Black alone and Hispanics have higher undercounts than the non-Hispanic White alone population. The fact that the same patterns were found in these three surveys and the decennial census reinforces the idea that these are real differences and not an artifact of a survey's methodology.

The undercount of young children in the census and in surveys has important implication for data on the characteristics of this population. First, the undercount in the census may lead to bias in the survey estimates because the Census Bureau controls the demographic surveys to the population estimates, which are based on the census. While the undercount of young children in the census could cause the population estimates for this age group to be biased downward (underestimated), the census coverage error will not affect the population estimates of children born after the census. Therefore, by 2005 or 2015, the population estimates for young children were no longer based on the census, but were developed primarily using data from vital statistics.

However, the undercount for young children in the decennial census will follow that cohort throughout the decade in the population estimates and could still potentially bias the survey estimates for older children as they age. We attempted to measure this cumulative coverage error - the combination of coverage error in the census base for the population estimates and coverage error in the surveys. The cumulative coverage error for young children would be greatest in the year of the census and the proceeding four years. In addition, the coverage errors from the census will be reproduced 
in the population estimates and will follow the cohort as it ages throughout the decade - which we clearly observed in the differences between the initial coverage rates and adjusted coverage rates for the 5 to 9 age group in the 2015 ACS and CPS.

Finally, the coverage error that we report here in the surveys could be an indicator of coverage bias in the survey statistics for young children [24]. Again, there is only coverage bias in the survey statistics when the characteristics of the population that is not covered by the survey are different from the population that is covered by the survey. In this report, we have shown that coverage rates for young children vary by race and Hispanic origin. To the extent that the differences between young children that are covered and not covered in the survey are correlated with other social and economic characteristics (e.g., poverty status or food insecurity), then these indicators for young children may also be biased. A different research design than we used here is needed to measure coverage bias in survey statistics caused by the undercount of this population in demographic surveys. However, it is clear from this analysis that in addition to having high net undercounts in the census, young children also have higher coverage error in demographic surveys than other age groups.

\section{Acknowledgments}

The authors would like to thank Scott Konicki, Debbie Griffin, Laurie Schwede, Bill O'Hare, and Heather King for their helpful comments on this manuscript. The views expressed are those of the authors and not necessarily those of the U.S. Census Bureau.

\section{References}

[1] Griffin DH. Final Task Force Report: Task Force on the Undercount of Young Children, Memorandum for Frank A. Vitrano, U.S. Census Bureau, Washington, DC. Issued February 2014.

[2] Coale AJ. The Population of the United States in 1950 Classified by Age, Sex, and Color - A Revision of Census Figures. Journal of the American Statistical Association. 1955; 50(269): 16-54.

[3] U.S. Census Bureau. 2020 Census Research and Testing: Investigating the 2010 Undercount of Young Children - A New Look at 2010 Census Omissions by Age. Issued July 26, 2016.

[4] Tourangeau R, Shapiro G, Kearney A, and Ernst L. Who lives here? Survey undercoverage and household roster questions. Journal of Official Statistics. 1997; 13(1): 1-18.

[5] Hogan H, Cantwell PJ, Devine J, Mule VT, and Velkoff V. Quality and the 2010 Census. Population Research and Policy Review. 2013; 32(5): 637-662.
[6] O'Hare WP, Robinson JG, West K, and Mule T. Comparing the US Decennial Census Coverage Estimates for Children from Demographic Analysis and Coverage Measurement Surveys. Population Research and Policy Review. 2016; 35(5): 685-704.

[7] O'Hare WP. The undercount of young children in the US Decennial Census. Springer International Publishing. 2015.

[8] West K, and Robinson JG. What Do We Know About The Undercount of Children? Population Division Working Paper No. 39. U. S. Census Bureau, Washington, DC 20233-8800. 1999.

[9] Durrant GB, and Steele F. Multilevel modelling of refusal and non-contact in household surveys: evidence from six UK Government surveys. Journal of the Royal Statistical Society: Series A (Statistics in Society). 2009; 172: 361-381.

[10] Groves RM, and Couper MP. Nonresponse in household survey interviews. New York: Wiley. 1998.

[11] U.S. Census Bureau. American Community Survey Design and Methodology. Washington, DC: U.S. Government Printing Office. 2014.

[12] U.S. Census Bureau. 2010 Census Planning Memoranda Series: 2010 Census Integrated Communications Program Paid Advertising Assessment Report. Washington, DC: U.S. Government Printing Office. 2012.

[13] Martin E Who knows who lives here? Within-household disagreements as a source of survey coverage error. Public Opinion Quarterly. 1999; 62(3): 220-236.

[14] Schwede L, Terry R, and Childs JH. Ethnographic Evaluations on Coverage of Hard-to-Count Minorities in U.S. Decennial Censuses (Chapter 14), in Hard-to-Survey Populations, edited by R. Tourangeau, Nancy Bates, Brad Edwards, Timothy Johnson, and Kirk Wolter. Cambridge University Press. 2014

[15] Schwede L, and Terry R. "Comparative Ethnographic Studies of Enumeration Methods and Coverage across Race/Ethnic Groups.” 2010 Census Program for Evaluations and Experiments Evaluation. 2010 Census Planning Memoranda Series, No. 255. March 29, 2013. Available from: http://www.census. gov/2010census/pdf/comparative_ethnographic_studies_of_ enumeration_methods_and_coverage_across_race_and_ ethnic_groups.pdf.

[16] Childs JH. "A Brief Review of Coverage, Ethnographic Studies, and Changing Census Bureau Operations since the 1970s," in Schwede, Laurie and Rodney Terry (2013), "Comparative Ethnographic Studies of Enumeration Methods and Coverage across Race/Ethnic Groups.” 2010 Census Program for Evaluations and Experiments Evaluation. 2010 Census Planning Memoranda Series, No. 255. March 29, 2013. Available from: http://www.census.gov/2010census/pdf/comparati ve_ethnographic_studies_of_enumeration_methods_and coverage_across_race_and_ethnic_groups.pdf.

[17] De la Puente M. Why are People Missed or Erroneously Enumerated in the Census: A Summary of Findings from Ethnographic Research. In Proceedings of the 1993 Research Conference on Undercounted Ethnic Populations. 1993; 29-66.

[18] Lin I, Schaeffer NC, Seltzer JA, and Tuschen KL. Divorced Parents' Qualitative and Quantitative Reports of Children's Living Arrangements. Journal of Marriage and Family. 2004; 66(2): 385-397.

[19] Waller MR, and Jones MR. Who is the residential parent? Understanding discrepancies in unmarried parents' reports. Journal of Marriage and Family. 2014; 76(1): 73-93.

[20] U.S. Census Bureau. Census Research and Testing: Investi- 
gating the 2010 Undercount of Young Children - Examining the Coverage of Young Mothers. Issued June 7, 2016.

[21] United States Federal Committee on Statistical Methodology. Measuring and Reporting Sources of Error in Surveys. Statistical Policy working Paper, No. 31. Washington, D.C.: United States Office of Management and Budget. 2001.

[22] U.S. Census Bureau. American Community Survey: Coverage Rates. 2016. Available from: http://www.census.gov/acs/ www/methodology/sample-size-and-data-quality/coveragerates/.

[23] Eckman S, and Kreuter F. Undercoverage rates and undercoverage bias in traditional housing unit listing. Sociological Methods \& Research. 2013; 42(3): 264-293.

[24] Groves RM, Fowler FJ, Couper MP, Lepkowski JM, Singer E and Tourangeau R. Survey methodology. John Wiley \& Sons. 2011.

[25] Loudermilk C, and Li M. A national evaluation of coverage for a sampling frame based on the Master Address File (MAF). Proceedings of the Section on Survey Research Methods, American Statistical Association, Washington, DC. 2009

[26] Hainer P, Hines C, Martin E, and Shapiro G. Research on Improving Coverage in Household Surveys. In Proceedings, Annual Research Conference. Washington, DC: Bureau of the Census. 1988

[27] Johnson K. The Hidden Cost of the Recession: Two Million Fewer Births and Still Counting. National Fact Sheet No. 26 (Durham, NH: Carsey School of Public Policy, University of New Hampshire). 2014.

[28] Scopilliti M, Bolender BC, and Knapp A. Cohort-Based Evaluation of the Vintage 2010 Population Estimates: The Role of International Migration 2000 to 2010. Presentation at the 2014 Population Association of America Annual Meeting. Boston, MA

[29] U.S. Census Bureau. Modified Race Summary File Methodology. Population Division. Updated: 07/05/2012. 2017. Available from: https://www2.census.gov/programs-surveys/ popest/technical-documentation/methodology/modified-racesummary-file-method/mrsf2010.pdf.
[30] Bennett C, and Griffin D. Race and Hispanic Origin Data: A Comparison of Results from the Census 2000 Supplementary Survey and Census 2000. 2002 Joint Statistical Meetings: Proceedings of the Section on Survey Research Methods. American Statistical Association.

[31] Raglin D, and Leslie T. How Consistent is Race Reporting Between the Census and the Census 2000 Supplementary Survey? 2002 Joint Statistical Meetings: Proceedings of the Section on Survey Research Methods. American Statistical Association.

[32] Jordan J, and Beaghen M. Analysis of the American Community Survey and Puerto Rico Community Survey Coverage, Decennial Statistical Studies Division, Washington, DC, USA, Memo. ACS13-RER-1, Jan. 16, 2013.

[33] del Pinal J, and Schmidley D. Matched Race and Hispanic Origin Responses from Census 2000 and Current Population Survey February to May 2000. Population Division Working Paper No. 79. Washington, DC: U.S. Census Bureau. 2005.

[34] U.S. Census Bureau. Current Population Survey-Design and Methodology, Technical Paper, No. 63RV, Washington DC: US Census Bureau. 2002.

[35] U.S. Census Bureau. Survey of Income and Program Participation (SIPP) 2008 Panel: Source and Accuracy Statement for Wave 1 to Wave 16 (core) Public Use Files (S\&A-18). 2015. Available from: https://www.census.gov/programs-surveys/ sipp/tech-documentation/source-accuracy-statements/sourceaccuracy-statements-2008.html.

[36] U.S. Census Bureau. Survey of Income and Program Participation: Weighting. 2016. Available from: https://www.census. gov/programs/surveys/sipp/methodology/weighting.html.

[37] Hogan HR, and Robinson JG. What the Census Bureau's Coverage Evaluation Programs Tell Us About Differential Undercount. Richmond, Va.: Bureau of the Census Research Conference on Undercounted Ethnic Populations. 1993.

[38] Jensen EB, Benetsky M, and Garrow S. Updating the 2010 Demographic Estimates of the Hispanic Population. Paper presented at the 2016 Annual Meetings of the Southern Demographic Society. Athens, GA. 